\title{
Influence of Parental Overweight on the Association of Birth Weight and Fat Distribution Later in Childhood
}

\author{
Amanda R.A. Adegboye ${ }^{a}$ Lars B. Andersen ${ }^{b, c}$ Niels Wedderkopp ${ }^{b}$ \\ Berit L. Heitmann ${ }^{a}$ b \\ ${ }^{a}$ Research Unit for Dietary Studies, Institute of Preventive Medicine, Copenhagen University \\ Hospital, Copenhagen, ${ }^{b}$ Center for Research in Childhood Health, Institute of Sports \\ Science and Clinical Biomechanics, University of Southern Denmark, Odense, Denmark, \\ 'Department of Sports Medicine, Norwegian School of Sport Sciences, Oslo, Norway
}

\section{Key Words}

Fetal development · Adiposity · Fat distribution - Children · Adolescents

\begin{abstract}
Objective: To examine whether the association between birth weight and fat distribution in childhood is modified by parental overweight. Methods: Cross-sectional study of 728 Danish children aged 8-10 and 14-16 years. The main outcomes were waist circumference, waist-toheight ratio, subscapular skinfold, and subscapular-to-triceps skinfold ratio. Analyses were stratified by parental overweight status (none vs. $\geq 1$ overweight parent) for each dependent variable, expressed as z-scores. Results: Birth weight z-score was negatively associated with waist circumference ( $\beta-0.08$ SD; $95 \%$ CI $-0.15,-0.02$ ), waist-to-height ratio $(\beta-0.15$ SD; $95 \%$ CI $-0.22,-0.07)$, and subscapular-to-triceps ratio ( $\beta-0.28$ SD; $95 \%$ CI $-0.44,-0.12)$ after adjustment for sex, age, puberty, preterm birth, BMI, height, socio-economic status, mother's age at delivery, parity, breastfeeding, energy intake, and aerobic fitness in the group with $\geq 1$ overweight parent. Birth weight was negatively associated with subscapular skinfold in groups with $(\beta-0.16$ SD; $95 \%$ CI $-0.24,-0.06)$ and without overweight parents $(\beta-0.09$ SD; $95 \%$ CI $-0.16,-0.02)$, but the magnitude of the association was greater in the former group. Conclusion: The association between birth weight and fat distribution seems to be influenced by parental overweight. Lower birth weights are associated with central adiposity among offspring of overweight parents.


Adegboye et al.: Influence of Parental Overweight on the Association of Birth

Weight and Fat Distribution Later in Childhood

\section{Introduction}

Fetal life has been suggested to be a critical period in relation to later adiposity [1]. Birth weight is frequently used as a marker of genetic factors and the intrauterine environment as it reflects underlying mechanisms influencing growth [2].

Some studies have found support for a linear relationship between birth weight and subsequent BMI development [3, 4], whereas others have found a J- or U-shaped association [5]. It has been suggested that J- or U-shaped associations might reflect a compensatory growth and fat accretion among individuals with low birth weight, which would result in an increased BMI [6]. However, this discrepancy in the association between birth weight and BMI may also depend on the fact that BMI does not distinguish between lean versus fat mass. Moreover, BMI fails to distinguish between central versus peripheral adiposity [2].

Although the link between impaired fetal development and low lean mass among children and adolescents seems well established, the relationship between birth weight and adiposity and fat distribution later in childhood is still unclear $[1,6]$. While some studies have shown a negative association between birth weight and central and total adiposity [7, 8], others have found a positive [4] or non-significant $[9,10]$ associations. This inconsistency may partly reflect differences in the assessments of adiposity and fat distribution and/or failure to control for important confounders.

In the past 10 years, a number of studies using accurate measures of adiposity, such as dual X-ray absorptiometry (DXA), have been conducted, but none of them had simultaneously accounted for parental BMI, breastfeeding, energy intake, and physical activity during childhood $[4,7,8,10-14]$. Since the impact of fetal conditions on later adiposity is likely to be attenuated by life courses, failure to adjust for actual lifestyle may result in spurious associations of birth weight and measures of adiposity or fat distribution. Also, familiar predisposition to obesity may influence this relationship, as offspring of obese parents have higher risk of obesity because of shared genes [15] and shared environments [16]. However, little is known about such influences.

The primary aim of this study was to examine whether the associations between birth weight and different measures of fat distribution, with comprehensive but sequential control for potential confounders, was modified by parental overweight. The secondary aim was to examine the same associations with BMI as a proxy for adiposity.

\section{Material and Methods}

The European Youth Heart Study (EYHS) is a longitudinal study of the associations between lifestyle and risk factors for cardiovascular diseases in schoolchildren, from which boys and girls in the 3rd (8-11 years) and 9th (14-16 years) grades were recruited in 1997/1998. Children in the 3rd grade were followed up after 6 years, and a new 3rd-grade cohort was selected. The data used in this study were restricted to information collected at baseline in Odense, Denmark, as part of the EYHS. The study was approved by the local ethics committee, and all children gave verbal consent and their parents written consent. Complete information on the cohort is presented elsewhere [17].

A total of 1,019 children participated in the study at baseline. The analysis was limited to Caucasian children whose biological parents completed the study questionnaires ( $\mathrm{n}=818)$. After exclusion of individuals with missing information on birth weight, gestational age, anthropometric measurements and parental BMI, the final sample consisted of 728 children and their parents.

No differences in sex, birth weight, and gestational age among participants and non-participants were observed. However, participation rate was higher among the 3rd-grade compared to the 9th-grade group. Participant parents were more likely to have higher educational level and less likely to be overweight compared to non-participant parents. 


\section{Variables}

Parents completed a questionnaire on their sociodemographic characteristics, lifestyle and current weight and height at baseline in 1997/1998. Education was defined as maternal high school degree (yes/ no). Paternal annual income was dived into low ( $<27,000$ EUR), medium (27,000-54,000 EUR) and high $\left(>54,000\right.$ EUR). Parental BMI was calculated as weight/height ${ }^{2}\left(\mathrm{~kg} / \mathrm{m}^{2}\right)$, using self-reported weight and height. Parents were also asked whether their child had ever been exclusively breastfed (yes/no) for at least 4 months. Further information on parity, birth weight, and gestational age was retrieved from the Danish Medical Birth Register. Maternal age at delivery was derived by subtracting the mother's date of birth from the child's date of birth.

Pubertal maturation was assessed according to Tanner staging [18], and classified as pre-pubertal (Tanner 1) versus pubertal/post-pubertal stage (Tanner 2-5). Information on dietary intake was obtained by a 24-hour recall, supported by a qualitative food record [19]. Total energy intake was introduced into the models as a continuous variable (kJ/day).

Aerobic fitness $\left(\mathrm{VO}_{2} \mathrm{max}\right)$ was assessed as the maximum power output in a cycle test, with progressively increasing workload on an electronically braked cycle ergometer (Monark 839 Ergomedic, Varberg, Sweden). Aerobic fitness was expressed as maximum power output relative to body weight (W/kg) [20]. Although aerobic fitness has a strong genetic component [21] in children; it has been shown to be positively associated with daily physical activity assessed by accelerometers, and is expressed in counts/min in this sample [20] and in other similar populations [22, 23]. Information on daily physical activity in counts/min was available only for a sub-sample of the study population $(n=465)$. Analyses were performed using the accelerometer data, but due low numbers and therefore low statistical power aerobic fitness was used instead.

Weight and height were measured with standard techniques using a beam-scale type weight and a stadiometer, respectively. BMI was calculated as weight $/$ height ${ }^{2}\left(\mathrm{~kg} / \mathrm{m}^{2}\right)$. Skinfold measurements (triceps, biceps, subscapular, and suprailiac) were taken on the left side of the body in duplicate or triplicate, according to the criteria described by Lohman et al. [24]. BMI and sum of 4 skinfold measurements were used as makers of adiposity. Waist circumference was measured midway between the lower rib margin and the iliac crest. Waist circumference, waist-to-height ratio, and subscapular skinfold were used as measurements of central adiposity. Subscapular-to-triceps ratio was calculated as indices of truncal to peripheral adiposity. Subscapular and triceps skinfolds were used because they are sites easy to locate and measure and therefore less vulnerable to measurement error [25].

Birth weight was used as a surrogate measure of intrauterine growth. Birth weight was expressed as the standard deviation (SD) from the expected birth weight (z-score) according to the sex-specific mean birth weight for gestational age based on a reference population [26]. Preterm birth was defined as birth before 37 weeks of gestation.

\section{Analysis}

All anthropometric indices, except for BMI, were standardized to internally derived z-scores, by sexand age-specific distribution, to account for the changes in variance by sex and age groups. BMI z-scores were calculated with reference to a Danish national sample [27].

Multiple linear regression models using robust standard errors, which accounted for clustering design (schools), were performed. Confounders were gradually introduced into the models: model 1 was adjusted for preterm birth, sex, age, and puberty; model 2 was adjusted for model 1 plus BMI and height (not applied for BMI analyses); model 3 was adjusted for model 2 plus maternal education and paternal income; model 4 was adjusted for model 3 plus mother's age at delivery and parity; and model 5 was adjusted for model 4 plus breastfeeding, energy intake, and aerobic fitness. Regression analyses including a quadratic term for birth weight, adjusted for all confounding factors, were also performed but evidence of non-linearity in any of the associations was not found.

BMI was examined as a binary outcome because birth weight may be associated with the upper end of the BMI distribution without affecting the mean. Hence, logistic regression was performed using overweight (yes/no) [28] as dependent variable and quartiles of birth weight z-scores as independent variables.

In order to investigate whether parental overweight (BMI $\geq 25 \mathrm{~kg} / \mathrm{m}^{2}$ ) influenced the association between birth weight and fat distribution and adiposity, all models were stratified by parental overweight status (no parental overweight vs. at least one overweight parent). The interaction between child's sex and 
Table 1. Characteristics of the study population ${ }^{\mathrm{a}}$

\begin{tabular}{|c|c|c|c|c|c|c|}
\hline \multirow[t]{3}{*}{ Characteristics } & \multicolumn{6}{|l|}{ EYHS } \\
\hline & \multicolumn{3}{|c|}{3 rd grade $(n=437 ; 8-11$ years $)$} & \multicolumn{3}{|c|}{9 th grade $(\mathrm{n}=291 ; 14-16$ years $)$} \\
\hline & all & girls & boys & all & girls & boys \\
\hline Age, years & $9.6(0.41)$ & $9.6(0.39)$ & $9.7(0.41)^{*}$ & $15.5(0.38)$ & $15.5(0.39)$ & $15.5(0.37)$ \\
\hline Birth weight, kg & $3.4(0.55)$ & $3.3(0.56)$ & $3.4(0.54)$ & $3.3(0.52)$ & $3.3(0.50)$ & $3.4(0.55)$ \\
\hline Gestational age, week & $39.6(1.9)$ & $39.6(2.0)$ & $39.5(1.8)$ & $39.8(1.6)$ & $39.9(1.6)$ & $39.6(1.7)$ \\
\hline Height, m & $1.39(0.06)$ & $1.39(0.06)$ & $1.40(0.06)$ & $1.70(0.07)$ & $1.66(0.06)$ & $1.75(0.07)^{*}$ \\
\hline BMI, $\mathrm{kg} / \mathrm{m}^{2}$ & $17.3(2.4)$ & $17.3(2.6)$ & $17.2(2.2)$ & $20.8(2.6)$ & $20.9(2.8)$ & $20.6(2.4)$ \\
\hline Waist circumference, $\mathrm{cm}$ & $58.6(5.9)$ & $58.4(6.1)$ & $58.8(5.6)$ & $69.8(6.1)$ & $68.2(6.0)$ & $71.6(5.9)^{*}$ \\
\hline Waist-to-height ratio & $0.42(0.04)$ & $0.42(0.04)$ & $0.42(0.03)$ & $0.41(0.03)$ & $0.41(0.03)$ & $0.41(0.03)$ \\
\hline Subscapular, mm & $8.7(5.3)$ & $9.7(5.8)$ & $7.4(4.3)^{*}$ & $11.5(5.4)$ & $13.6(6.0)$ & $9.2(3.6)^{*}$ \\
\hline Subscapular-to-triceps ratio & $0.75(0.27)$ & $0.82(0.32)$ & $0.68(0.18)^{*}$ & $0.96(0.33)$ & $0.99(0.37)$ & $0.92(0.26)$ \\
\hline Sum of skinfolds, $\mathrm{mm}$ & $37.7(19.0)$ & $40.7(19.8)$ & $34.3(17.6)^{*}$ & $45.5(20.2)$ & $53.4(19.9)$ & $37.1(16.8)^{*}$ \\
\hline
\end{tabular}

${ }^{a}$ Values presented are means (SD). Comparisons between girls and boys in each school grade group.

*Significant difference $\mathrm{p}<0.05$

birth weight z-scores was tested. Since no evidence was found that the strength of the association differed between sexes, analyses were not performed separately for boys and girls. Statistical analyses were performed using STATA version 9.2 (Statacorp LP, College Station, TX, USA).

\section{Results}

Table 1 shows the population characteristics according to sex and age groups. In the 3rd-grade group, there was a small, albeit significant, gender difference in age, in that girls were slightlyyounger than boys. Girls in the 3rd grade had a higher mean value of subscapularto-triceps ratio compared to boys. In the 9th-grade, boys were taller and had higher mean values of waist circumference compared to girls. However, waist-to-height ratio did not differ between genders. Girls in both groups had higher mean values of sum of 4 skinfolds measurements than boys. Girls started puberty earlier than boys. In the 3rd-grade group, $29 \%$ of girls had already entered puberty, whereas all boys were at pre-pubertal stage. In the 9 th grade group, all girls had started puberty and $3 \%$ of boys were still at pre-pubertal stage (data not shown).

In total, $27.3 \%$ of mothers and $46.3 \%$ of fathers had BMI $\geq 25 \mathrm{~kg} / \mathrm{m}^{2}$. Overall, $57 \%$ of the children had at least one overweight parent, and $16 \%$ of the children had two overweight parents. Overweight parents were more likely to have overweight children (65\%) than nonoverweight parents (35\%; $\mathrm{p}<0.0001)$.

\section{Fat Distribution}

Table 2 presents results regarding fat distribution. In the crude model, birth weight was positively related to waist circumference in children and adolescents regardless of parental overweight status, but the association was statistically significant only among those with at least one overweight parent. The direction of the association changed when total adiposity (BMI z-score) and body size (height z-score) were added to the model in both groups (model 2). In the fully adjusted model (model 5), birth weight was negatively associated with waist circumference among offspring of overweight parents; equivalent to -0.08 SDs $(95 \%$ CI 


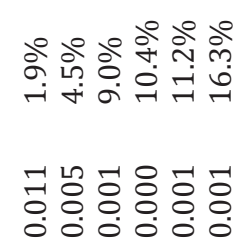

응조옹ㅇํㅇ

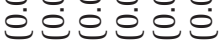
눈 논 î

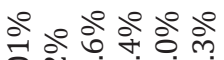

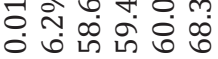

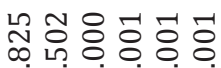

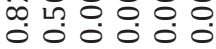

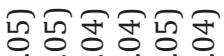
e.

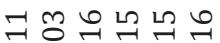
i 100000

ํํㅇํํํํำ ㅇำ ○ 궁 तु

๓

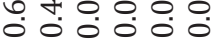

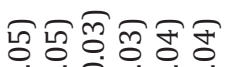

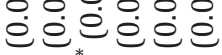

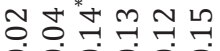
页 1000.

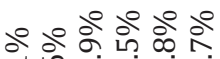

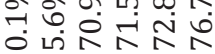

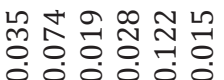
드ำตำ च०० 0 包包

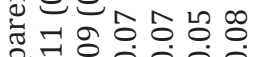
先 5

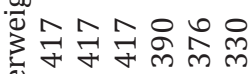

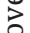

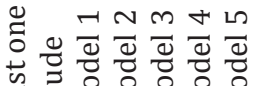

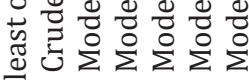
茹

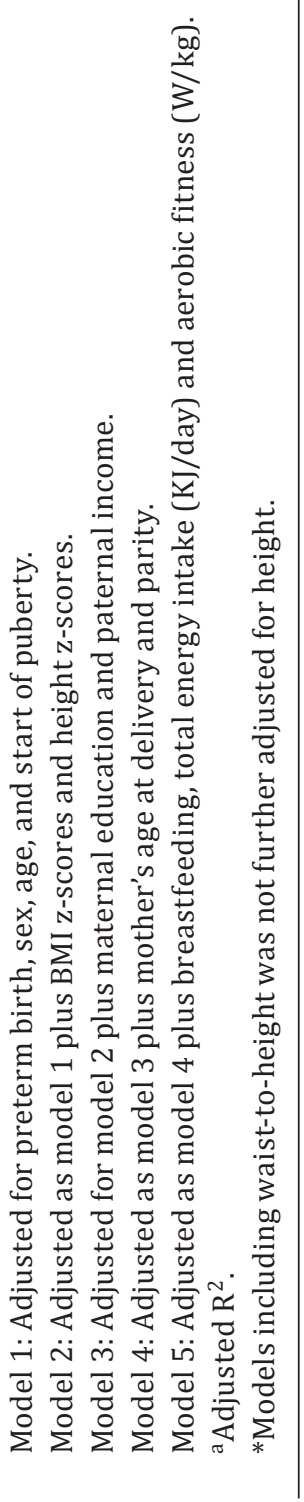


$-0.15,-0.02)$ change in waist circumference per 1 SD increase in birth weight. No significant associations were found among offspring of non-overweight parents.

In the crude model, birth weight was negatively associated with weight-to-height ratio later in childhood, but the association was not statistically significant in any of the groups related to parental overweight. Adjustment for confounders strengthened the magnitude of the associations, but only in the group with at least one overweight parent. In fact, the association between birth weight and weight-to-height ratio was 7 times greater in the fully adjusted model (model 5) compared to the crude model (from coefficient $=-0.02, p=0.683$ to coefficient $=-0.15, \mathrm{p}<0.001$ ). On the contrary, in the group with non-overweight parents the association between birth weight and weight-to-height ratio was not significant, and adjustment for confounders attenuated the magnitude of the associations (from a coefficient of $-0.91, \mathrm{p}=0.10$ to $-0.06, \mathrm{p}=0.148$ ).

There was a significant negative association between birth weight and subscapular skinfold in the fully adjusted models regardless of parental overweight status. However, the association was stronger in the group with overweight parents (coefficient $=-0.16,95 \% \mathrm{CI}$ $-0.24,-0.06$ ) compared to the group of non-overweight parents (coefficient $=-0.09,95 \% \mathrm{CI}$ $-0.16,-0.02)$.

In the crude model, there was a significant negative association between birth weight and subscapular-to-triceps ratio later in childhood in the group with at least one overweight parent, only. Adjustment for confounders strengthened the magnitude of the association (from coefficient $=-0.16, p=0.011$ to coefficient $=-0.28, p=0.001$ ). In the group with nonoverweight parents, adjustment for confounders did not influence the magnitude of the association, and associations remained non-significant in all models.

Including physical activity as counts/min, available in a sub-sample of the study population, did not markedly change the associations between birth weight and any of the fat location indices (waist circumference, waist-to-height ratio, subscapular skinfold, and subscapular-to-triceps ratio) in the final models (results not shown).

\section{Adiposity}

As regards adiposity, no significant associations were found between birth weight and BMI in the group with non-overweight parents, irrespective of adjustment for confounders (table 3). In the group with at least one overweight parent, birth weight was significantly and positively associated with BMI after adjustment for sex, age, puberty, maternal education, paternal income, mother's age at delivery, and parity. Birth weight was no longer associated with BMI when lifestyle (e.g. breastfeeding, current energy intake, and aerobic fitness) was taken into account. Among the three factors investigated, aerobic fitness had the major influence on the attenuation of the association. Interestingly, the association remained significant when breastfeeding and energy intake were added to the model (coefficient = $0.14, p=0.033$, adjusted $R^{2}=9.6 \%$ ). However, the association was completely lavished when fitness was added to the model. Inclusion of fitness increased the adjusted $\mathrm{R}^{2}$ from $9.6 \%$ to $36.4 \%$.

When using BMI as a binary variable, the likelihood of being overweight in childhood was significantly higher in the second $(\mathrm{OR}=1.83,95 \% \mathrm{CI} 1.04,3.21)$, third $(\mathrm{OR}=1.76,95 \%$ CI 1.07, 3.09) and fourth (OR $=2.23,95 \% \mathrm{CI} 1.28,3.89$ ) quartile compared to the first quartile of birth weight in the group with at least one overweight parent. The associations remained significant until breastfeeding and total energy intake were introduced into the model, and the associations were completely lavished after inclusion of aerobic fitness (data not shown). In the group with non-overweight parents, the associations were non-significant in both crude and fully adjusted models. Birth weight was not associated with sum of four skinfolds regardless of parental overweight status (data not shown). 
Table 3. Estimate of changes in BMI in z-scores per $1 \mathrm{SD}$ increase in birth weight among schoolchildren according to parental overweight status

\begin{tabular}{lllll}
\hline Models & $\mathrm{N}$ & \multicolumn{2}{l}{ BMI } & \\
\cline { 3 - 5 } & & $\beta(\mathrm{SE})$ & $\mathrm{p}$ & $\mathrm{R}^{2 \mathrm{a}}$ \\
\hline No parental overweight & & & & \\
Crude & 311 & $-0.002(0.06)$ & 0.973 & 0 \\
Model 1 & 311 & $0.02(0.06)$ & 0.790 & $7.2 \%$ \\
Model 2 & 311 & $-0.001(0.06)$ & 0.981 & $7.4 \%$ \\
Model 3 & 291 & $0.002(0.06)$ & 0.975 & $8.6 \%$ \\
Model 4 & 286 & $0.02(0.06)$ & 0.738 & $27.8 \%$ \\
\hline At least one overweight parent & & & \\
Crude & 417 & $0.15(0.05)$ & 0.006 & $1.8 \%$ \\
Model 1 & 417 & $0.13(0.05)$ & 0.015 & $8.3 \%$ \\
Model 2 & 390 & $0.14(0.06)$ & 0.012 & $8.6 \%$ \\
Model 3 & 376 & $0.13(0.06)$ & 0.039 & $8.8 \%$ \\
Model 4 & 330 & $0.06(0.05)$ & 0.262 & $36.4 \%$ \\
\hline
\end{tabular}

Model 1: Adjusted for preterm birth, sex, age and start of puberty.

Model 2: Adjusted as model 1 plus maternal education and paternal income.

Model 3: Adjusted as model 2 plus mother's age at delivery and parity.

Model 4: Adjusted as model 3 plus breastfeeding, total energy intake (kJ/day) and aerobic fitness (W/kg).

${ }^{\text {a Adjusted }} \mathrm{R}^{2}$.

Including physical activity as counts/min did not alter the association between birth weight and BMI or sum of four skinfolds in the final models (data not shown).

\section{Discussion}

The present study shows that parental overweight, as a marker for familial predisposition to obesity, noticeably influenced the association between birth weight and fat distribution later in childhood. Among offspring of overweight parents, those with lower birth weight z-scores had stored more fat centrally than those with higher birth weight z-scores for all anthropometric indices investigated. These associations were independent of sex, age, puberty, BMI, height, maternal education, paternal income, mother's age at delivery, parity, breastfeeding, energy intake, and aerobic fitness. Among offspring of non-overweight parents, there was a significant association of birth weight and later subscapular skinfold, only. However, the magnitude of the association was smaller compared to the group with overweight parents.

To the best of our knowledge, none of the previous studies investigating the role of fetal growth on fat distribution and adiposity later in child hood had simultaneously stratified the analysis by parental overweight status and controlled for post-natal and current lifestyle. Thus, it is difficult to fully compare our results with others. However, our findings seems consistent with the hypothesis that familiar predisposition to obesity has a great effect on offspring fat distribution. A study examined the contributions of genetic factors and the family environment to human fatness in a sample of adult Danish adoptees and found that there was a strong relation between the body weight of the adoptees and the BMI of their biological parents, but no relation to their adoptive parents. The study concluded that 
genetic influences have an important role in determining human fatness in adults, whereas the family environment alone has no apparent effect [15]. Nevertheless, in the present study the possibility of confounding, as overweight parents may provide a different social environment for their offspring than non-overweight parents, cannot be totally excluded.

Albeit a significant positive association between birth weight and BMI later in childhood in the group with at least one overweight parent was evident, the association was abolished after adjustment for aerobic fitness. It remains uncertain whether this lack of association between birth weight and adiposity may be explained in part by the use of crude measures of adiposity such as BMI. Conversely, a previous study found that girls with high birth weight, low fitness level, and at least one parent with overweight were significantly more likely to be classified as overweight/obese at age 12-18 years [29]. However, important confounders such as sexual maturation, gestational age, and dietary factors were not taken into account.

Inconsistent results have been reported even when adiposity was measured by 4-component model in two earlier studies [11,12]. One study showed that birth weight was not associated with fat mass index and percentage fat among children, adolescents, and young adults in the analyses either unadjusted or after adjustment for puberty, physical activity, socioeconomic status, ethnicity, and parental BMI [11], while another study showed that a higher birth weight was significantly associated with lower percentage fat later in childhood (6.5-9.1 years) after adjustment for age, sex, socioeconomic status, physical activity, BMI, and body shape [12]. However, it is also possible that using crude information on physical activity and failure to adjust for breastfeeding and energy intake during childhood might have obscured the true association between birth weight and total adiposity in these particular studies.

Study limitations warrant some thoughts. Studies involving children and adolescents are of great importance because they are old enough for the amplification of programming effects but not so old as to impose an expressive contribution of lifestyle factors, which cumulatively influence the body composition and health status of older subjects [10]. This cohort was composed of children with a wide age range, from 8 to 16 years, which introduces some methodological challenges in that older children have been exposed for a longer period of time to a larger variety of environmental factors than younger children. However, we were able to control for many lifestyle characteristics, including energy intake and fitness, that may contribute to total adiposity and fat distribution.

Sexual maturation is another challenge because body dimension may change rapidly during puberty. Although it was possible to control the analyses for pubertal stage, age and height, information on peak height velocity (growth spurt) was not available, and therefore the possibility of residual confounding cannot be excluded further.

Skinfold thickness is not considered a highly accurate measurement of fat distribution, and waist circumference does not distinguish between intraabdominal and subcutaneous abdominal fat. Nevertheless, waist circumference is associated with cardiovascular risk factors in pre- and post-pubertal children [30]. Highly sophisticated, but expensive methods such as computed tomography and magnetic resonance imaging could add more information on the role of fetal weight in the development of intraabdominal fat in children. In the present study, the use of more crude measures (waist circumference) may have led to attenuation of the observed associations. Hence, true associations may be expected to be even stronger.

It was not possible to use cutpoints for definition of low birth weight $(<2.5 \mathrm{~kg})$ or small for gestational age (SGA), and macrosomia ( $>4 \mathrm{~kg}$ ) to aid comparison of effect size with previous studies, due to the low number of low birth weight, SGA, and macrosomia in our population. Therefore, birth weight, expressed as z-score, in the range of normal distribution was used instead. 
Parental weight was self-reported and consequently subject to underreporting, particularly among overweight/obese individuals [31]. A population-based study conducted in $1999 / 2001$ reported $60 \%$ and $43.6 \%$ of overweight/obesity among 30- to 60 -year-old Danish men and women, respectively [32]. Similarly, in this study overweight was higher among fathers than mothers; however, the prevalence was lower $(46.3 \%$ in fathers and $27.3 \%$ in mothers) than estimates reported previously [33]. Such underreporting would lead to misclassification of overweight parents into normal-weight parents and hence results in an attenuation of any differences seen in the associations for the non-overweight and the overweight groups. However, the strength of the associations was already markedly different between groups, supporting the trust in our findings.

The dose-response effect was also explored by performing stratified analyses for those with normal weight, overweight and obese parents. Although there was a trend towards higher effect sizes (magnitude of the associations) among children with obese parents compared to those with overweight or normal-weight parents for all outcomes investigated, the analyses were markedly influenced by the lack of statistical power. In our sample, 84 children had at least one obese parent, and only 12 had two obese parents. Therefore, some results did not reach statistical significance, and some estimates showed wide confidence intervals. The comparison between children with one overweight parent and those with two overweight parents showed effect sizes of essentially similar magnitude.

Despite the potential limitations, this is one of only a few studies with extensive information on potential pre- and post-natal determinants of fat distribution and obesity in childhood, including direct measurement of birth weight and gestational age, and objective measurements of children food intake, and physical fitness.

The association between birth weight and fat distribution later in childhood was conventionally significant, but the magnitude of the fully adjusted effect size was rather modest. Obesity is notoriously difficult to treat and for that reason early identification of children at a high risk for becoming overweight may offer the best opportunity for a target-preventive effort. In this scenario, although the association between birth weight and fat distribution may be modest, size at birth combined with other established risk factors, particularly overweight/obesity among their parents can be used as a potential screening tool in primary obesity interventions.

\section{Conclusion}

The association between birth weight and fat distribution later in childhood seems markedly influenced by parental overweight. Lower birth weights were associated with central adiposity among offspring of overweight parents, even after control for a wide range of confounders.

\section{Acknowledgment}

We acknowledge all members of the EYHS group, particularly Karsten Forberg, Niels C. Møller, Peter Lund Christensen for making their data available. The study received financial support from the TRYG foundation.

\section{Disclosure Statement}

The authors declare no conflict of interest. 
Adegboye et al.: Influence of Parental Overweight on the Association of Birth

Weight and Fat Distribution Later in Childhood

\section{References}

1

$>2$

$>3$

2008; $16: 2524-2528$.

4 Rogers IS, Ness AR, Steer CD, Wells JC, Emmett PM, Reilly JR, Tobias J, Smith GD: Associations of size at birth and dual-energy X-ray absorptiometry measures of lean and fat mass at 9 to $10 \mathrm{y}$ of age. Am J Clin Nutr 2006; 84:739-747.

5 Parsons TJ, Power C, Manor 0: Fetal and early life grow th and body mass index from birth to early adulthood in 1958 British cohort: longitudinal study. BMJ 2001;323:1331-1335.

- 6 Rogers I: The influence of birthweight and intrauterine environment on adiposity and fat distribution in later life. Int J Obes Relat Metab Disord 2003;27:755-777.

- 7 Dolan MS, Sorkin JD, Hoffman DJ: Birth weight is inversely associated with central adipose tissue in healthy children and adolescents. Obesity (Silver Spring) 2007;15:1600-1608.

- 8 Labayen I, Ruiz JR, Vicente-Rodriguez G, Turck D, Rodriguez G, Meirhaeghe A, Molnar D, Sjostrom M, Castillo MJ, Gottrand F, Moreno LA: Early life programming of abdominal adiposity in adolescents: The HELENA Study. Diabetes Care 2009;32:2120-2122.

9 Euser AM, Finken MJ, Keijzer-Veen MG, Hille ET, Wit JM, Dekker FW: Associations between prenatal and infancy weight gain and BMI, fat mass, and fat distribution in young adulthood: a prospective cohort study in males and females born very preterm. Am J Clin Nutr 2005;81:480-487.

$\checkmark 10$ Singhal A, Wells J, Cole TJ, Fewtrell M, Lucas A: Programming of lean body mass: a link between birth weight, obesity, and cardiovascular disease? Am J Clin Nutr 2003;77:726-730.

11 Chomtho S, Wells JC, Williams JE, Lucas A, Fewtrell MS: Associations between birth weight and later body composition: evidence from the 4-component model. Am J Clin Nutr 2008;88:1040-1048.

12 Elia M, Betts P, Jackson DM, Mulligan J: Fetal programming of body dimensions and percentage body fat measured in prepubertal children with a 4-component model of body composition, dual-energy X-ray absorptiometry, deuterium dilution, densitometry, and skinfold thicknesses. Am J Clin Nutr 2007;86:618624.

13 Fewtrell MS, Lucas A, Cole TJ, Wells JC: Prematurity and reduced body fatness at 8-12 y of age. Am J Clin Nutr 2004;80:436-440.

14 Labayen I, Moreno LA, Blay MG, Blay VA, Mesana MI, Gonzalez-Gross M, Bueno G, Sarria A, Bueno M: Early programming of body composition and fat distribution in adolescents. J Nutr 2006;136:147-152.

-15 Stunkard AJ, Sorensen TI, Hanis C, Teasdale TW, Chakraborty R, Schull WJ, Schulsinger F: An adoption study of human obesity. N Engl J Med 1986;314:193-198.

-16 Kalakanis LE, Goldfield GS, Paluch RA, Epstein LH: Parental activity as a determinant of activity level and patterns of activity in obese children. Res Q Exerc Sport 2001;72:202-209.

17 Riddoch C, Edwards D, Page A, Froberg K, Andersen SA, Wedderkoop N, Brage S, Cooper A, Sardinha LB, Harro M, Heggebø LK, Mechelen WV, Borehan C, Ekelund U, Andersen LB, European Youth Heart Study Team: The European Youth Heart Study - cardiovascular disease risk factors in children: rationale, aims, study design and validation of methods. J Phys Activ Health 2005;2:115-129.

18 Tanner JM: Growth and maturation during adolescence. Nutr Rev 1981;39:43-55.

19 Nielsen BM, Bjornsbo KS, Tetens I, Heitmann BL: Dietary glycaemic index and glycaemic load in Danish children in relation to body fatness. Br J Nutr 2005;94:992-997.

$\checkmark 20$ Andersen LB, Harro M, Sardinha LB, Froberg K, Ekelund U, Brage S, Anderssen SA: Physical activity and clustered cardiovascular risk in children: a cross-sectional study (The European Youth Heart Study). Lancet 2006;368:299-304.

21 Bray MS, Hagberg JM, Perusse L, Rankinen T, Roth SM, Wolfarth B, Bouchard C: The human gene map for performance and health-related fitness phenotypes: the 2006-2007 update. Med Sci Sports Exerc 2009;41: 35-73.

22 Dencker M, Thorsson 0, Karlsson MK, Linden C, Svensson J, Wollmer P, Andersen LB: Daily physical activity and its relation to aerobic fitness in children aged 8-11 years. Eur J Appl Physiol 2006;96:587-592.

23 Eiberg S, Hasselstrom H, Gronfeldt V, Froberg K, Svensson J, Andersen LB: Maximum oxygen uptake and objectively measured physical activity in Danish children 6-7 years of age: the Copenhagen school child intervention study. Br J Sports Med 2005;39:725-730.

24 Lohman TG, Roche AF, Martorell R: Anthropometric Standardization Reference Manual. Champaign, Human Kinetics, 1991.

25 Ruiz L, Colley JR, Hamilton PJ: Measurement of triceps skinfold thickness. An investigation of sources of variation. Br J Prev Soc Med 1971;25:165-167.

26 Kramer MS, Platt RW, Wen SW, Joseph KS, Allen A, Abrahamowicz M, Blondel B, Breart G: A new and improved population-based Canadian reference for birth weight for gestational age. Pediatrics 2001; 108:E35. 
27 Nysom K, Molgaard C, Hutchings B, Michaelsen KF: Body mass index of 0 to 45-y-old Danes: reference values and comparison with published European reference values. Int J Obes Relat Metab Disord 2001;25:177184.

28 Adegboye AR, Andersen LB, Froberg K, Sardinha LB, Heitmann BL: Linking definition of childhood and adolescent obesity to current health outcomes. Int J Pediatr Obes 2010;5:130-142.

29 Vale S, Santos R, Soares-Miranda L, Mota J: The relationship of cardiorespiratory fitness, birth weight and parental BMI on adolescents' obesity status. Eur J Clin Nutr 2010;64:622-627.

30 Garnett SP, Baur LA, Srinivasan S, Lee JW, Cowell CT: Body mass index and waist circumference in midchildhood and adverse cardiovascular disease risk clustering in adolescence. Am J Clin Nutr 2007;86: 549-555.

31 Dijkshoorn H, Ujcic-Voortman JK, Viet L, Verhoeff AP, Uitenbroek DG: Ethnic variation in validity of the estimated obesity prevalence using self-reported weight and height measurements. BMC Public Health 2011; 11:408.

-32 Pisinger C, Jorgensen T: Weight concerns and smoking in a general population: the Inter99 study. Prev Med 2007;44:283-289.

33 Bendixen H, Holst C, Sorensen TI, Raben A, Bartels EM, Astrup A: Major increase in prevalence of overweight and obesity between 1987 and 2001 among Danish adults. Obes Res 2004;12:1464-1472. 